\title{
Identification and characterisation of microRNAs in young adults of Angiostrongylus cantonensis via a deep-sequencing approach
}

\author{
Shih-Hsin Chang ${ }^{1}$ Petrus Tang ${ }^{1,2,3}$, Cheng-Hung Lai ${ }^{4}$, Ming-Ling Kuo ${ }^{1,5}$, Lian-Chen Wang ${ }^{1,2} /+$ \\ ${ }^{1}$ Graduate Institute of Biomedical Sciences ${ }^{3}$ Bioinformatics Center ${ }^{2}$ Department of Parasitology \\ ${ }^{5}$ Department of Microbiology and Immunology, College of Medicine, Chang Gung University, Kwei-Shan, Taoyuan County, Taiwan \\ ${ }^{4}$ Department of Veterinary Medicine, College of Veterinary Medicine, National Chung Hsing University, Taichung City, Taiwan
}

\begin{abstract}
Angiostrongylus cantonensis is an important causative agent of eosinophilic meningitis and eosinophilic meningoencephalitis in humans. MicroRNAs (miRNAs) are small non-coding RNAs that participate in a wide range of biological processes. This study employed a deep-sequencing approach to study miRNAs from young adults of A. cantonensis. Based on 16,880,456 high-quality reads, 252 conserved mature miRNAs including 10 antisense miRNAs that belonging to 90 families, together with 10 antisense miRNAs were identified and characterised. Among these sequences, 53 miRNAs from 25 families displayed 50 or more reads. The conserved miRNA families were divided into four groups according to their phylogenetic distribution and a total of nine families without any members showing homology to other nematodes or adult worms were identified. Stem-loop real-time polymerase chain reaction analysis of aca-miR-1-1 and aca-miR-71-1 demonstrated that their level of expression increased dramatically from infective larvae to young adults and then decreased in adult worms, with the male worms exhibiting significantly higher levels of expression than female worms. These findings provide information related to the regulation of gene expression during the growth, development and pathogenesis of young adults of A. cantonensis.
\end{abstract}

Key words: Angiostrongylus cantonensis - deep-sequencing approach -

microRNA - stem-loop real-time polymerase chain reaction - young adults

\begin{abstract}
Angiostrongylus cantonensis, the rat lungworm, is the primary causative agent of eosinophilic meningitis and eosinophilic meningoencephalitis in humans (RamirezAvila et al. 2009). This species was first discovered in rats from China in 1935 (Chen 1935) and the first human case was reported in Taiwan in 1945 (Nomura \& Lin 1945). Although this infection is mainly prevalent in Southeast Asia and the Pacific Basin (Asato et al. 2004, Hochberg et al. 2011, Qu et al. 2011, Tsai et al. 2011), many new cases have been reported among travellers returning from endemic areas (Bärtschi et al. 2004, Ali et al. 2008, Malvy et al. 2008, Luessi et al. 2009) and, by 2008, more than 2,800 cases had been recorded worldwide (Wang et al. 2008). The major clinical manifestations of this disease include headache, neck stiffness, muscle weakness, nausea, vomiting and paraesthesia. Although the clinical course of infection is usually benign and self-limited, severe infections may lead to irreversible outcomes and even death (Punyagupta et al. 1975).

The life cycle of $A$. cantonensis is complex, requiring both an intermediate host and a final definitive host. The
\end{abstract}

doi: 10.1590/0074-0276108062013005

Financial support: NSC, Executive Yuan, ROC (NSC96-2320-B-

182-020, NSC100-2320-B-182-013), Chang Gung Memorial Hospital

Research (CMRPD150121, CMRPD1A0111)

+ Corresponding author:wanglc@mail.cgu.edu.tw

Received 22 November 2012

Accepted 13 June 2013 giant African snail Achatina fulica and the channelled apple snail Pomacea canaliculata are the two most common intermediate hosts for A. cantonensis worldwide (Kliks \& Palumbo 1992, Lv et al. 2009). Following entry into the intermediate host through the oral or percutaneous route, the first-stage larvae undergo two moults and develop into the infective larvae within two-three weeks. Humans are an accidental host for this parasite, becoming infected with the parasite by ingesting raw or uncooked individuals of intermediate host species ( $A$. $f u$ lica, $P$. canaliculata, Hyla aurea), paratenic host species (Macrobrachium lar, Ocypode ceratophthalma, Hoplobatrachus rugulosus, Rana plancyi, Tilapia mossambica, Vaginulus plebeius, Laevicaulis alte) (freshwater crustaceans, frogs, fish and planarians) or contaminated vegetables carrying the third-stage larvae (Wallace \& Rosen 1966, 1967, Ash 1968, Tsai et al. 2004). These larvae then penetrate the intestinal tract and migrate to the central nervous system, where they moult twice and develop into young adults, approximately one week after infection (Alicata 1965).

The available genomic information for A. cantonensis is limited to data from small-scale transcriptomic investigations and cloning studies. Following the submission of 1,226 expressed sequence tags (ESTs) from young adult $A$. cantonensis (5th-stage larva) to the National Center for Biotechnology Information dbEST by our laboratory in 2005, these and some additional sequences were analysed and were found to encode proteins participating in metabolism, cellular development, immune evasion and host-parasite interactions ( $\mathrm{Xu}$ et al. 2009) and were subsequently grouped into 13 categories (Fang et al. 2010). Among proteins encoded by cDNA 
clusters found in the fourth-stage larvae, an enrichment of binding and catalytic activity was also revealed (He et al. 2009). The transcripts found in pepsin-activated infective larvae (3rd-stage) encode proteins that participate in a wide range of biological processes (Chang et al. 2011). Although cloning of galectin (Hao et al. 2007), cystatin (Liu et al. 2010), Ac16 (Li et al. 2011), cathepsin B (Han et al. 2011), a cathepsin B-like cysteine proteinase (Cheng et al. 2012) and a protein disulphide isomerase (Liu et al. 2012) has been accomplished in $A$. cantonensis, the characterisation of these proteins is far from complete. Moreover, the genome of this parasite has not yet been sequenced.

MicroRNAs (miRNAs) are a type of small noncoding RNAs, approximately $21-23$ nucleotides (nt) in length, that have been discovered in diverse organisms, including animals, plants, viruses and fungi (Ambros 2004, Lee et al. 2010, Khan et al. 2011, Plaisance-Bonstaff \& Renne 2011). Because the first two miRNAs, lin-4 and let-7, were discovered and demonstrated to be involved in the regulation of developmental timing in Caenorhabditis elegans (Lee et al. 1993, Reinhart et al. 2000), more than 15,000 microRNA gene loci in over 140 species have been reported in miRBase v16 (Kozomara \& Griffiths-Jones 2011). MiRNAs regulate a wide range of biological processes, including development, metabolism, cell proliferation and differentiation (Bartel 2009, Krol et al. 2010). In animals, miRNAs usually silence their target mRNAs through degradation or translational repression (Nilsen 2007). With respect to experimental methods, computational approaches are considered a useful strategy for identifying miRNAs, even in species whose genomes have not been fully sequenced (Berezikov et al. 2006). Computational approaches can also be utilised to quantify miRNA expression (Luo 2012).

As parasitic helminths usually exhibit a complex life cycle, investigations involving miRNAs enable us to not only understand the roles of these riboregulators in the physiology, development and evolution of these organisms, but also the mechanisms of host invasion and pathogenesis (Xue et al. 2008, Hao et al. 2010, Poole et al. 2010, Chen et al. 2011a, Winter et al. 2012). MiRNA expression in adult worms of $A$. cantonensis was recently identified and compared between the sexes (Chen et al. 2011b). The adult worms may only cause severe obstruction of the pulmonary arteries and respiratory failure (Wang et al. 2012). In contrast, because the young adults of $A$. cantonensis are at a pathogenic developmental stage associated with the central nervous system, miRNA expression profiling may provide further information on the pathogenesis of eosinophilic meningitis and eosinophilic meningoencephalitis. In the present study, we employed a deep-sequencing approach to identify conserved miRNAs among young adults of $A$. cantonensis. Following a phylogenic distribution analysis, the expression profiles of selected miRNAs at different developmental stages were analysed via stem-loop real-time polymerase chain reaction (RT-PCR).

\section{MATERIALS AND METHODS}

Parasites - A strain of A. cantonensis has been maintained in our laboratory in Biomphalaria glabrata snails and Sprague-Dawley rats since 1980 (Wang et al. 1989). Young adults and adult worms were collected on days 21 and 50 post-infection, respectively, from the cerebral tissues and pulmonary arteries of rats. The sex of these worms was determined based on their morphological characteristics: male worms are usually shorter and exhibit copulatory bursa and long spicules. Infective larvae were collected from the tissues of infected snails through digestion with $0.6 \%(\mathrm{w} / \mathrm{v})$ pepsin- $\mathrm{HCl}(\mathrm{pH} 2-3)$ for $1 \mathrm{~h}$. These worms were washed with normal saline, phosphate buffered saline and distilled water and then stored at $-80^{\circ} \mathrm{C}$ for further analyses (Hwang et al. 2010). This experiments performed in this study followed the recommendations of the Institutional Animal Care and Use Committee of Chang Gung University.

Small RNA library construction and high-throughput sequencing - Total RNA was isolated from young adults of $A$. cantonensis (500 worms of each sex) using the TRI Reagent, according to the instructions of the manufacturer (Molecular Research Center, Cincinnati, $\mathrm{OH}, \mathrm{USA})$. RNA integrity was assessed via $1 \%(\mathrm{w} / \mathrm{v})$ agarose gel electrophoresis, while RNA purity was determined based on the absorbance recorded at 260/280 nm using an SMA1000 UV Spectrophotometer (Merinton Technology, Beijing, China). The RNA preparations were then stored at $-80^{\circ} \mathrm{C}$ for further experiments.

RNA fragments of 18-30 nt in length were separated from total RNA using the Small RNA Sample Prep Kit (Illumina, San Diego, CA, USA). Following 15\% Novex TBE-urea polyacrylamide gel electrophoresis (PAGE), the purified fragments were ligated to $5^{\prime}$ and $3^{\prime}$ RNA adaptors, reverse transcribed to produce single-stranded cDNAs and amplified via PCR. RNA fragments (approximately $92 \mathrm{bp}$ ) were isolated from the PCR products and sequenced using the Illumina HiSeq 2000 system (Illumina, San Diego, CA, USA).

Bioinformatic analysis - The small RNA library was analysed through a deep-sequencing small RNA analysis pipeline (DSAP) (dsap.cgu.edu.tw) (Huang et al. 2010). Following the removal of adaptors and poly-A/T/C/G/N $\mathrm{nt}$, sequences longer than $16 \mathrm{nt}$ were considered clean sequence tags. Using the clustering module of DSAP, redundant clean reads were merged into unique tags. The reads for each unique tag were counted as its expression abundance.

To identify transfer RNAs (tRNAs), ribosomal RNAs (rRNAs), small nucleolar RNAs (snoRNAs), small nuclear RNAs (snRNAs) and other annotated noncoding RNAs (ncRNAs), the unique tags were subjected to searches against the transcribed sequence library of the ncRNA (Rfam) database (version 10.0). The remaining sequences were compared with mature miRNA sequences in miRBase (version 16) using BLASTN.

Stem-loop RT-PCR - Total RNAs were isolated from infective larvae (20,000 worms), young adults (500 worms of each sex) and adult worms (50 worms of each 
sex). The expression levels of aca-miR-1-1 and aca-miR71-1 were determined via modified stem-loop RT-PCR (Chen et al. 2005). The stem-loop reverse transcription primer and forward primer for aca-miR-1-1 were 5'-GTCGTATCCAGTGCAGGGTCCGAGGTATTCGCACTGGATACGACTACATAC-3' and 5'-CGCGGCTGGAATGTAAAGAAGT-3', respectively, and those for aca-miR-71-1 were 5'-GTCGTATCCAGTGCAGGGTCCGAG-GTATTCGCACTGGATACGACCGTCTCA-3' and 5'-GCGCGGTGAAAGACATGG-GTAGTG-3', respectively. The reverse primer employed in these assays was 5'-GTGCAGGGTCCGAGGT-3'.

Small RNAs from A. cantonensis at different developmental stages were extracted using an miRNA isolation kit (Geneaid, Sijhih City, Taipei County, Taiwan) according to the instructions of the manufacturer. First-strand cDNAs were synthesised using SuperScript III Reverse Transcriptase (Invitrogen, Carlsbad, CA, USA). Briefly, reaction mixtures $(20 \mu \mathrm{L})$ containing the purified small RNAs (160 ng), 5X First-Strand buffer (4 $\mu \mathrm{L})$, individual specific stem-loop RT primers $(0.5 \mu \mathrm{M})$, dNTP mix (0.5 mM), DTT (5 mM), SuperScript III Reverse Transcriptase (10 units) and RNaseOUT (2 units) were incubated at $55^{\circ} \mathrm{C}$ for $50 \mathrm{~min}$, then inactivated by heating at $70^{\circ} \mathrm{C}$ for $15 \mathrm{~min}$ and subsequently incubated with two units of RNase $\mathrm{H}$ at $37^{\circ} \mathrm{C}$ for $20 \mathrm{~min}$.

Quantitative RT-PCR was performed using RealQ PCR Master Mix (Ampliqon A/S, Skovlunde, Denmark) in the Stratagene Mx3000P QPCR System (Agilent Technologies, Santa Clara, CA, USA). The PCR mixtures (20 $\mu \mathrm{L})$ included the reverse transcription product for each miRNA $(3 \mu \mathrm{L})$, RealQ-PCR $2 X$ Master Mix $(10 \mu \mathrm{L})$, a specific forward primer $(0.5 \mu \mathrm{M})$ and the reverse primer $(0.5 \mu \mathrm{M})$. The RT-PCR assays were carried out with an initial step at $95^{\circ} \mathrm{C}$ for $10 \mathrm{~min}$, followed by 40 cycles of amplification, with denaturation at $95^{\circ} \mathrm{C}$ for $30 \mathrm{~s}$, primer annealing at $58^{\circ} \mathrm{C}$ for $1 \mathrm{~min}$ and elongation at $72^{\circ} \mathrm{C}$ for 30 s. The specificity of this assay was confirmed via $15 \%$ PAGE. Finally, the expression levels of the targeted miRNAs were determined through three rounds of stem-loop RT-PCR amplification and analysed according to the $2^{-\Delta \Delta \mathrm{Ct}}$ method (Livak \& Schmittgen 2001).

Statistical analysis - The expression levels of the miRNAs were expressed as the mean \pm standard deviation. Means were compared via one-way ANOVA and the least significance difference test was used for posthoc multiple range comparisons. $\mathrm{p}<0.05$ was considered to be statistically significant.

\section{RESULTS}

Analysis of short RNAs - A total of 22,484,156 raw sequence reads were obtained through high-throughput sequencing from the small RNA library generated for young adults of $A$. cantonensis. The removal of adapters, contaminated nt and low-quality sequences resulted in $16,880,456$ (75.1\%) high-quality, clean reads of 16-31 nt in length, averaging $22.8 \mathrm{nt}$. A majority of the sequences were $23 \mathrm{nt}$ in length (46.1\%), followed by lengths of $22 \mathrm{nt}$ (26.3\%) and $24 \mathrm{nt}(12.1 \%)$ sequences. Among the clean reads, $10,766,590(63.8 \%)$ sequences were found to match entries in the Rfam database. They included 7,736,727 tRNAs (45.8\%), 1,191,869 rRNAs (7.1\%), 228,262 snoRNAs (1.4\%), 174,633 snRNAs (1\%) and 1,435,099 ncRNAs $(8.5 \%)$ (Fig. 1).

Identification of conserved miRNAs - From the remaining 7,548,965 clean reads, 252 conserved mature miRNAs including 10 antisense miRNAs were identified based on comparison with entities in miRbase. All of the identified miRNAs were homologous to miRNAs from Metazoa and their lengths ranged from 20-24 nt, with most being $22 \mathrm{nt}$ in length (117), followed by lengths of $21 \mathrm{nt}$ (54), $23 \mathrm{nt}$ (49), $20 \mathrm{nt}$ (19) and $24 \mathrm{nt}$ (13). These miRNAs belonged to 90 families, in addition to which 10 antisense miRNAs were discovered. The number of reads obtained was 50 or more for 53 of these miRNAs, 21-49 for 28 miRNAs, $11-20$ for 14 miRNAs, two-10 for 82 miRNAs and only a single read was observed in the remaining 75 miRNAs.

Supplementary data lists the 53 miRNAs with 50 or more reads, which belonged to 25 families. More than one miRNA was identified from the following families: let-7, mir-1, mir-34, mir-87, mir-71 mir-99, mir-2, mir-9, mir-31, mir-50 and mir-103. Only one miRNA was found in the remaining 14 families. Nine miRNAs displayed more than 1,000 reads. Aca-miR-1-1 exhibited the highest number of reads, at 184,946 , followed by aca-miR71-1 $(92,433)$. These two highly expressed miRNAs constituted $50.7 \%$ and $25.3 \%$ of the total reads $(365,112)$, respectively, among this group of 53 miRNAs.

Phylogenic distribution - To analyse the evolutionary features of $A$. cantonensis miRNAs, the identified miRNAs showing 50 or more reads, from 25 families, were compared with those from other nematodes in miRBase. The families let-7, mir-1, mir-2, mir-9, mir-31, mir34, mir-44, mir-50, mir-67, mir-71, mir-81, mir-87 and mir-124 contained members that were homologous to parasitic nematodes (Ascaris suum and Brugia malayi) and free-living nematodes (C. elegans, Caenorhabditis

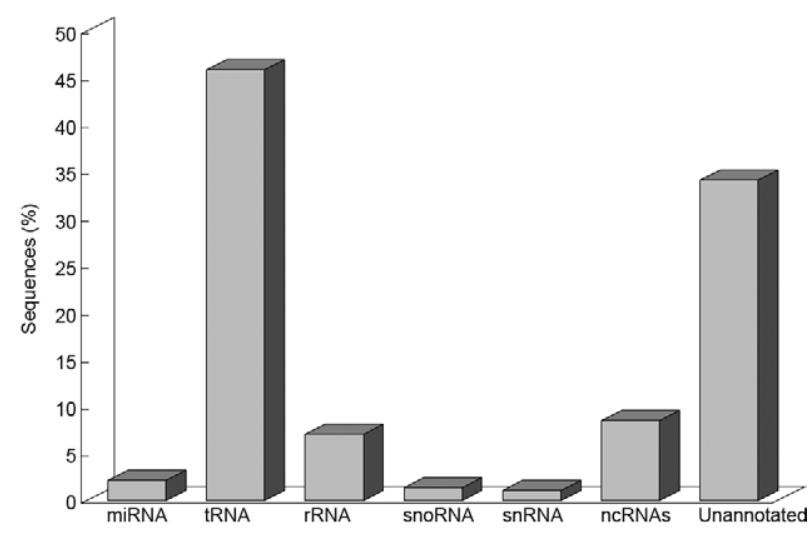

Fig. 1: classification of small RNAs of Angiostrongylus cantonensis young adults identified by a deep-sequencing approach. miRNA: microRNAs; ncRNA: non-coding RNAs; rRNA: ribosomal RNAs; snoRNA: small nucleolar RNAs; snRNA: small nuclear RNAs; tRNA: transfer RNAs. 
briggsae, Caenorhabditis remanei and Pristionchus pacificus). Members of the mir-60 and mir-235 families were homologous to sequences from free-living nematodes, but not parasitic nematodes. The family mir-99 was homologous only to A. suum, while members of the mir-21, mir-29, mir-30, mir-103, mir-140, mir-146, mir185, mir-191 and mir-320 families did not exhibit homology with sequences from other nematodes (Table).

Expression of miRNAs at different developmental stages - To confirm the expression of the miRNAs from $A$. cantonensis young adults that were identified through the applied high-throughput approach and to determine the expression levels of these miRNAs at different developmental stages, the levels of aca-miR-1-1 and aca-miR71-1 transcripts were validated in a modified stem-loop quantitative RT-PCR analysis. Significant differences in the expression levels of these miRNAs were observed among different developmental stages (aca-miR-1-1: $\mathrm{F}=$ 521.55, $\mathrm{p}<0.001$; aca-miR-71-1: $\mathrm{F}=1585.86, \mathrm{p}<0.001)$. In male worms, the expression of the two miRNAs was significantly higher in young adults than in infective larvae or adult worms $(p<0.05)$. Additionally, the expression in adult worms was significantly higher than in infective larvae $(\mathrm{p}<0.05)$. In female worms, significantly higher expression was also observed in young adults $(\mathrm{p}<0.05)$. However, there was no significant difference in aca-miR1-1 expression observed between infective larvae and adult worms $(p>0.05)$ and the infective larvae exhibited significantly higher aca-miR-71-1 expression than adult worms $(p<0.05)$. Moreover, male worms displayed significantly higher expression than female worms in both the young adult and adult stages $(\mathrm{p}<0.05)$ (Fig. 2).

\section{DISCUSSION}

Using a deep-sequencing approach, based on $7,548,965$ reads, we identified and characterised 252 conserved mature miRNAs including 10 antisense miRNAs that belonging to 90 families from young adults of A. cantonensis. Previous authors identified miRNAs in adult male and female worms from 592,899 and 458,447 reads, respectively (Chen et al. 2011b). However, no novel miRNAs were discovered in either the present or previous studies. The sequences obtained from the adult male and female worms were matched to the C. elegans genome, although the percentage of perfect matches was quite low (18.94\% in females and $22.58 \%$ in males). Although C. elegans and A. cantonensis are both nematodes, the former species is free-living in soil or water, whereas the latter is parasitic, being found in the pulmonary arteries of its definitive host and requiring a molluse as an intermediate host (Chen et al. 2011b). In the present study, a search for sequences in miRBase discovered only conserved mature miRNAs. We hypothesise that novel miRNAs will be identified only when a reference genome for A. cantonensis becomes available.

In the present study, we identified nine miRNAs in young adults of $A$. cantonensis displaying more than a 1,000 reads: two in the let- 7 family, four in the mir-1 family, one in the mir-44 family, one in the mir-71 family and one in the mir-99 family. In male adult worms, seven miRNAs exhibited more than 1,000 reads: miR-1,
miR-228, miR-44, miR-45, miR-71, miR-72 and miR-81. In the female worms, 10 miRNAs showed more than a 1,000 reads: miR-1, miR-2, miR-228, miR-44, miR-45, miR-60, miR-71, miR-72, miR-81 and miR-87 (Chen et al. 2011b). MirR-1, mirR-71 and miR-44 show high expression in both the young adult and in adult stages. In contrast, let-7 and miR-99 are highly expressed only in young adults, whereas miR-45 and miR- 81 are expressed only in adult worms. These findings indicate that a stage-specific expression of miRNAs occurs in $A$. cantonensis. Moreover, the regulatory functions of miR99 require further investigation.

Let-7 and lin-4 are two important miRNA families associated with the lifespan of $C$. elegans. These miRNAs have been characterised as playing an essential role in the developmental timing of the worm by downregulating specific targets, such as the TRIM protein lin-41 and the transcription factor lin-14 (Ibáñez-Ventoso et al. 2006, Ambros 2011). Let-7 is an miRNA families that was discovered in ancient animals (Christodoulou et al. 2010). The let-7 family is expressed in a wide range of animals and the sequences of its members are highly conserved. However, significant variations in the size of the let-7 family occur among organisms: of the 55 organisms known to express members of the let-7 family, 13 express only one let-7 miRNA, including the nematodes $P$. pacificus, C. remanei, C. elegans, C. briggsae and B. malayi, whereas 19 mature let-7 sequences have been identified in the zebrafish (Danio rerio) (Pasquinelli et al. 2003). In the present study, we identified 19 members of the let-7 family in young adults of A. cantonensis, 11 of which exhibited 50 or more reads. In adult worms, the copy number of this miRNA was determined to be less than 50 in both sexes. These finding suggests the importance of let-7 in gene regulation in young adults of $A$. cantonensis. However, understanding the role of let-7 in the development of this parasite will require further studies.

Among the miRNA families found to be expressed in young adults of $A$. cantonensis, mir-1 displayed the highest number of total reads. Members of this family have been found in a wide range of organisms, including worms, flies, fishes, mice and humans (Bentwich et al. 2005, Sokol \& Ambros 2005, Wienholds et al. 2005, Zhao et al. 2005). They are evolutionarily conserved and have been characterised as playing essential roles in regulating proliferation and the differentiation of muscle development via the regulation of synaptic transmission (Simon et al. 2008, Jones et al. 2011). In adult worms, the mir-71 family presented the highest number of reads in both sexes (Chen et al. 2011b). This family has been reported to promote longevity and stress resistance in worms (Pincus et al. 2011) and is involved in the sexual maturation of female worms (Gomes et al. 2011). These expression patterns suggest the different roles of miRNAs at different developmental stages.

Although the phylogenetic distribution has been reported for Clonorchis sinensis (Xu et al. 2010), there is no such information available for parasitic nematodes. The 25 conserved miRNA families found in A. cantonensis young adults showed a distribution bias. These conserved families can be divided into four groups: 13 
families were homologous to sequences of other parasitic nematodes, two to sequences of free-living nematodes, but not parasitic nematodes, and one to A. suum sequences. Nine families did not exhibited any member that was homologous to either a free-living or parasitic nematode. Moreover, these miRNAs observed in young adults of $A$. cantonensis were not found in adult worms (Chen et al. 2011b). It is possible that the first two groups regulate general biological or physiological functions in nematodes. The miRNAs showing homology to $A$. suum may be specific to parasitic nematodes. As adult worms of $A$. cantonensis live within the central nervous system, the last group may regulate adaptive functions of worms related to this special environment, which may also cause pathological changes in the central nervous system. Further studies are required to confirm these hypotheses.

Analysis of the levels of aca-miR-1-1 and aca-miR71-1 expression via the stem-loop RT-PCR revealed different expression patterns based on developmental stages and sex. These two miRNAs were selected because they are highly expressed not only in young adults, but also in adult worms of both sexes. In both male and female worms, the level of expression of these miRNAs increased dramatically from the levels observed in infective larvae and peaked in young adults, subsequently declining to a low level in adult worms. Overall, the expressions levels of these miRNAs were found to be highest in male adult males. Similar expression patterns have been reported for 18 miRNAs in adult worms (Chen et al. 2011b). These miRNAs may be important in regulating sex differentiation, rather than developmental stages. The lower expression levels of these miRNAs observed in female worms indicate that females may require a lower degree of post-transcriptional regulation than male worms. Moreover, the higher expression levels of the miRNAs detected in young adults suggest that more significant changes may occur during the young adult stage than in the adult stage.

Replicate analyses were difficult in the present study because of the technical difficulties involved in obtaining sufficient sample sizes from infected animals. This limitation may restrict the quantitation of our findings. However, we identified 53 miRNAs, belonging to 25 families, that displayed 50 or more reads. These findings provide reliable information about the global miRNA expression profiles found in A. cantonensis. Al-

TABLE

Phylogenic distributions of conserved microRNAs families of Angiostrongylus cantonensis young adults with 50 or more reads

\begin{tabular}{|c|c|c|c|c|c|c|c|}
\hline Family & A. cantonensis & Ascaris suum & Brugia malayi & $\begin{array}{c}\text { Caenorhabditis } \\
\text { elegans }\end{array}$ & $\begin{array}{c}\text { Caenorhabditis } \\
\text { briggsae }\end{array}$ & $\begin{array}{c}\text { Caenorhabditis } \\
\text { remanei }\end{array}$ & $\begin{array}{c}\text { Pristionchus } \\
\text { pacificus }\end{array}$ \\
\hline let-7 & + & + & + & + & + & + & + \\
\hline $\operatorname{mir}-1$ & + & + & - & + & - & + & + \\
\hline mir-2 & + & + & + & + & - & + & - \\
\hline mir-9 & + & + & + & + & + & + & + \\
\hline $\operatorname{mir}-21$ & + & - & - & - & - & - & - \\
\hline $\operatorname{mir}-29$ & + & - & - & - & - & - & - \\
\hline $\operatorname{mir}-30$ & + & - & - & - & - & - & - \\
\hline $\operatorname{mir}-31$ & + & - & + & - & - & + & + \\
\hline $\operatorname{mir}-34$ & + & + & + & + & + & + & - \\
\hline $\operatorname{mir}-44$ & + & + & - & + & + & + & + \\
\hline $\operatorname{mir}-50$ & + & + & + & + & + & + & - \\
\hline $\operatorname{mir}-60$ & + & - & - & + & + & + & - \\
\hline $\operatorname{mir}-67$ & + & + & - & + & + & + & - \\
\hline $\operatorname{mir}-71$ & + & + & + & + & + & + & - \\
\hline $\operatorname{mir}-81$ & + & + & - & + & + & + & - \\
\hline $\operatorname{mir}-87$ & + & + & + & + & + & + & + \\
\hline mir-99 & + & + & - & - & - & - & - \\
\hline mir-103 & + & - & - & - & - & - & - \\
\hline mir-124 & + & + & + & + & + & + & + \\
\hline mir-140 & + & - & - & - & - & - & - \\
\hline mir-146 & + & - & - & - & - & - & - \\
\hline mir-185 & + & - & - & - & - & - & - \\
\hline mir-191 & + & - & - & - & - & - & - \\
\hline $\operatorname{mir}-235$ & + & - & - & + & - & + & - \\
\hline $\operatorname{mir}-320$ & + & - & - & - & - & - & - \\
\hline
\end{tabular}




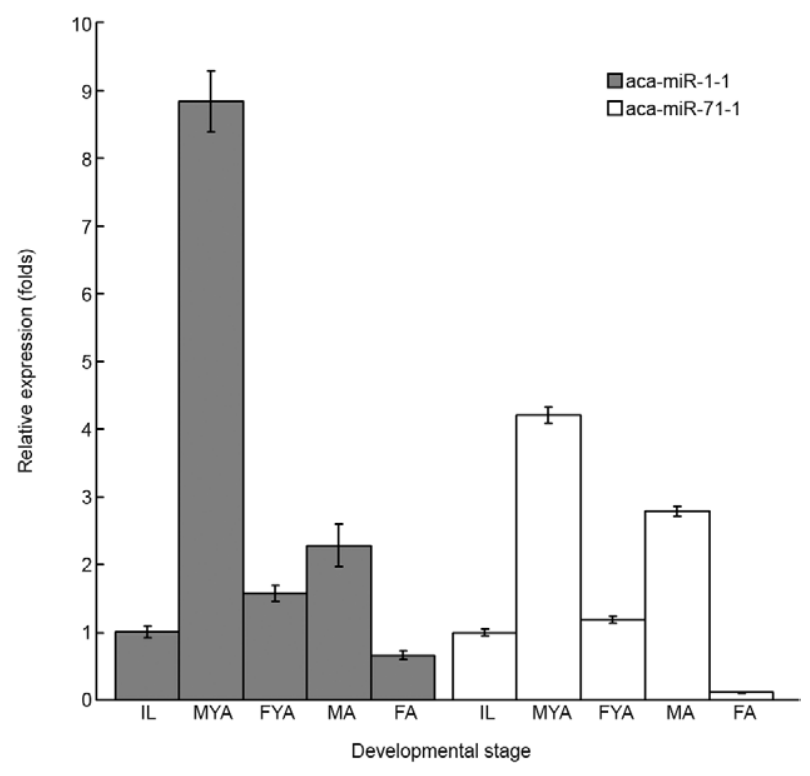

Fig. 2: expression profiles of selected microRNAs of Angiostrongylus cantonensis young adults in different developmental stages. FA: female adults; FYA: female young adults; IL: infective larvae; MA: male adults; MYA: male young adults.

though Northern blotting is considered a gold-standard approach for detecting miRNAs, this method is limited by its low sensitivity and difficulties in distinguishing homologous miRNAs from highly similar sequences (van Rooij 2011, Pritchard et al. 2012). Moreover, we succeeded in confirming the expression of two miRNAs initially identified via the high-throughput approach in A. cantonensis young adults through the more sensitive and specific technique of stem-loop quantitative RTPCR. These findings demonstrated the reliability of the results obtained herein.

Based on the results of the present study, there are significant differences in the expression of miRNAs between young adults and adult worms of A. cantonensis. These differences are not only qualitative, but also quantitative. In the present study, we identified nine miRNA families without homologous members in the available sequences of other nematodes in the adult stage. Moreover, the expression levels of miR-1 and miR-71 increase from a low expression level in infective larvae to a peak in young adults and subsequently decrease in adult worms. These results suggest that miRNAs play a more important role in the regulation of biological functions in young adults than in adult worms of $A$. cantonensis.

\section{REFERENCES}

Ali AB, Van den Enden E, Van Gompel A, Van Esbroeck M 2008. Eosinophilic meningitis due to Angiostrongylus cantonensis in a Belgian traveller. Travel Med Infect Dis 6: 41-44.

Alicata JE 1965. Biology and distribution of the rat lungworm, Angiostrongylus cantonensis and its relationship to eosinophilic meningoencephalitis and other neurological disorders of man and animals. Adv Parasitol 3: 223-248.
Ambros V 2004. The functions of animal microRNAs. Nature 431: 350-355.

Ambros V 2011. MicroRNAs and developmental timing. Curr Opin Genet Dev 21: 511-517.

Asato R, Taira K, Nakamura M, Kudaka J, Itokazu K, Kawanaka M 2004. Changing epidemiology of angiostrongyliasis cantonensis in Okinawa prefecture, Japan. Jpn J Infect Dis 57: 184-186.

Ash LR 1968. The occurrence of Angiostrongylus cantonensis in frogs of New Caledonia with observations on paratenic hosts of metastrongyles. J Parasitol 54: 432-436.

Bartel DP 2009. MicroRNAs: target recognition and regulatory functions. Cell 136: 215-233.

Bärtschi E, Bordmann G, Blum J, Rothen M 2004. Eosinophilic meningitis due to Angiostrongylus cantonensis in Switzerland. Infection 32: 116-118.

Bentwich I, Avniel A, Karov Y, Aharonov R, Gilad S, Barad O, Barzilai A, Einat P, Einav U, Meiri E, Sharon E, Spector Y, Bentwich $\mathrm{Z} 2005$. Identification of hundreds of conserved and nonconserved human microRNAs. Nat Genet 37: 766-770.

Berezikov E, Cuppen E, Plasterk RH 2006. Approaches to microRNA discovery. Nat Genet 38 (Suppl.): S2-S7.

Chang SH, Tang P, Wang LC 2011. A transcriptomic study on the pepsin-activated infective larvae of Angiostrongylus cantonensis. Mol Biochem Parasitol 179: 47-50.

Chen C, Ridzon DA, Broomer AJ, Zhou Z, Lee DH, Nguyen JT, Barbisin M, Xu NL, Mahuvakar VR, Andersen MR, Lao KQ, Livak KJ, Guegler KJ 2005. Real-time quantification of microRNAs by stem-loop RT-PCR. Nucleic Acids Res 33: e179.

Chen HT 1935. Un nouveau nematode pulmonaire, Pulmonem A. cantonensis n.g., n. sp. des rats de Canton. Ann Parasitol 13: 312-317.

Chen MX, Ai L, Xu MJ, Chen SH, Zhang YN, Guo J, Cai YC, Tian LG, Zhang LL, Zhu XQ, Chen JX 2011a. Identification and characterization of microRNAs in Trichinella spiralis by comparison with Brugia malayi and Caenorhabditis elegans. Parasitol Res 109: 553-558.

Chen MX, Ai L, Xu MJ, Zhang RL, Chen SH, Zhang YN, Guo J, Cai YC, Tian LG, Zhang LL, Zhu XQ, Chen JX 2011b. Angiostrongylus cantonensis: identification and characterization of microRNAs in male and female adults. Exp Parasitol 128: 116-120.

Cheng M, Yang X, Li Z, He H, Qu Z, He A, Wu Z, Zhan X 2012. Cloning and characterization of a novel cathepsin B-like cysteine proteinase from Angiostrongylus cantonensis. Parasitol Res 110: 2413-2422.

Christodoulou F, Raible F, Tomer R, Simakov O, Trachana K, Klaus S, Snyman H, Hannon GJ, Bork P, Arendt D 2010. Ancient animal microRNAs and the evolution of tissue identity. Nature 463: 1084-1088.

Fang W, Xu S, Wang Y, Ni F, Zhang S, Liu J, Chen X, Luo D 2010. ES proteins analysis of Angiostrongylus cantonensis: products of the potential parasitism genes? Parasitol Res 106: 1027-1032.

Gomes MS, Muniyappa MK, Carvalho SG, Guerra-Sá R, Spillane C 2011. Genome-wide identification of novel microRNAs and their target genes in the human parasite Schistosoma mansoni. Genomics 98: 96-111.

Han YP, Li ZY, Li BC, Sun X, Zhu CC, Ling XT, Zheng HQ, Wu ZD, Lv ZY 2011. Molecular cloning and characterization of a cathepsin B from Angiostrongylus cantonensis. Parasitol Res 109: 369-378.

Hao L, Cai P, Jiang N, Wang H, Chen Q 2010. Identification and characterization of microRNAs and endogenous siRNAs in Schistosoma japonicum. BMC Genomics 11: 55. 
Hao L, Wu K, Chen XG, Wang Q 2007. Cloning, prokaryotic expression and immunoreactivity evaluation of Angiostrongylus cantonensis galectin. Nan Fang Yi Ke Da Xue Xue Bao 27: 584-587.

He H, Cheng M, Yang X, Meng J, He A, Zheng X, Li Z, Guo P, Pan Z, Zhan X 2009. Preliminary molecular characterization of the human pathogen Angiostrongylus cantonensis. BMC Mol Biol 10: 97.

Hochberg NS, Blackburn BG, Park SY, Sejvar JJ, Effler PV, Herwaldt BL 2011. Eosinophilic meningitis attributable to Angiostrongylus cantonensis infection in Hawaii: clinical characteristics and potential exposures. Am J Trop Med Hyg 85: 685-690.

Huang PJ, Liu YC, Lee CC, Lin WC, Gan RR, Lyu PC, Tang P 2010. DSAP: deep-sequencing small RNA analysis pipeline. Nucleic Acids Res 38: W385-W391.

Hwang KP, Chang SH, Wang LC 2010. Alterations in the expression level of a putative aspartic protease in the development of $\mathrm{An}$ giostrongylus cantonensis. Acta Trop 113: 289-294.

Ibáñez-Ventoso C, Yang M, Guo S, Robins H, Padgett RW, Driscoll M 2006. Modulated microRNA expression during adult lifespan in Caenorhabditis elegans. Aging Cell 5: 235-246.

Jones AK, Rayes D, Al-Diwani A, Maynard TP, Jones R, Hernando G, Buckingham SD, Bouzat C, Sattelle DB 2011. A Cys-loop mutation in the Caenorhabditis elegans nicotinic receptor subunit UNC-63 impairs but does not abolish channel function. $J$ Biol Chem 286: 2550-2558.

Khan GA, Declerck M, Sorin C, Hartmann C, Crespi M, LelandaisBrière C 2011. MicroRNAs as regulators of root development and architecture. Plant Mol Biol 77: 47-58.

Kliks MM, Palumbo NE 1992. Eosinophilic meningitis beyond the Pacific Basin: the global dispersal of a peridomestic zoonosis caused by Angiostrongylus cantonensis, the nematode lungworm of rats. Soc Sci Med 34: 199-212.

Kozomara A, Griffiths-Jones S 2011. miRBase: integrating microRNA annotation and deep-sequencing data. Nucleic Acids Res 39: D152-D157.

Krol J, Loedige I, Filipowicz W 2010. The widespread regulation of microRNA biogenesis, function and decay. Nat Rev Genet 11: 597-610.

Lee HC, Li L, Gu W, Xue Z, Crosthwaite SK, Pertsemlidis A, Lewis ZA, Freitag M, Selker EU, Mello CC, Liu Y 2010. Diverse pathways generate microRNA-like RNAs and Dicer-independent small interfering RNAs in fungi. Mol Cell 38: 803-814.

Lee RC, Feinbaum RL, Ambros V 1993. The C. elegans heterochronic gene lin- 4 encodes small RNAs with antisense complementarity to lin-14. Cell 75: 843-854.

Li ZY, Lv ZY, Wei J, Liao Q, Zheng HQ, Wu ZD 2011. Cloning and characterization of a novel gene encoding $16 \mathrm{kDa}$ protein (Ac16) from Angiostrongylus cantonensis. Parasitol Res 110: 2145-2153.

Liu Q, Yang X, Zhang M, Wang L, Liu J, Chen J, He A, Li Z, Wu Z, Zhan X 2012. Molecular characterization and immunolocalization of a protein disulfide isomerase from Angiostrongylus cantonensis. Parasitol Res 110: 2501-2507.

Liu YH, Han YP, Li ZY, Wei J, He HJ, Xu CZ, Zheng HQ, Zhan XM, Wu ZD, Lv ZY 2010. Molecular cloning and characterization of cystatin, a cysteine protease inhibitor, from Angiostrongylus cantonensis. Parasitol Res 107: 915-922.

Livak KJ, Schmittgen TD 2001. Analysis of relative gene expression data using real-time quantitative PCR and the 2(-Delta Delta C(T)) Method. Methods 25: 402-408.

Luessi F, Sollors J, Torzewski M, Müller HD, Siegel E, Blum J, Sommer C, Vogt T, Thömke F 2009. Eosinophilic meningitis due to Angiostrongylus cantonensis in Germany. J Travel Med 16: 292-294.
Luo S 2012. MicroRNA expression analysis using the Illumina microRNA-Seq Platform. Method Mol Biol 822: 183-188.

Lv S, Zhang Y, Liu HX, Hu L, Yang K, Steinmann P, Chen Z, Wang LY, Utzinger J, Zhou XN 2009. Invasive snails and an emerging infectious disease: results from the first national survey on $A n-$ giostrongylus cantonensis in China. PLoS Negl Trop Dis 3: e368.

Malvy D, Ezzedine K, Receveur MC, Pistone T, Crevon L, Lemardeley P, Josse R 2008. Cluster of eosinophilic meningitis attributable to Angiostrongylus cantonensis infection in French policemen troop returning from the Pacific Islands. Travel Med Infect Dis 6: 301-314.

Nilsen TW 2007. Mechanisms of microRNA-mediated gene regulation in animal cells. Trends Genet 23: 243-249.

Nomura S, Lin PH 1945. First case report of human infection with Hamostrongylus ratti Yokogawa. Formosan Medical World 3: 589-592.

Pasquinelli AE, McCoy A, Jiménez E, Saló E, Ruvkun G, Martindale MQ, Baguñà J 2003. Expression of the 22 nucleotide let-7 heterochronic RNA throughout the Metazoa: a role in life history evolution? Evol Dev 5: 372-378.

Pincus Z, Smith-Vikos T, Slack FJ 2011. MicroRNA predictors of longevity in Caenorhabditis elegans. PLoS Genet 7: e1002306.

Plaisance-Bonstaff K, Renne R 2011. Viral miRNAs. Method Mol Biol 721: 43-66.

Poole CB, Davis PJ, Jin J, McReynolds LA 2010. Cloning and bioinformatic identification of small RNAs in the filarial nematode, Brugia malayi. Mol Biochem Parasitol 169: 87-94.

Pritchard CC, Cheng HH, Tewari M 2012. MicroRNA profiling: approaches and considerations. Nat Rev Genet 13: 358-369.

Punyagupta S, Juttijudata P, Bunnag T 1975. Eosinophilic meningitis in Thailand. Clinical studies of 484 typical cases probably caused by Angiostrongylus cantonensis. Am J Trop Med Hyg 24: 921-931.

Qu ZY, Yang X, Cheng M, Lin YF, Liu XM, He A, Wu ZD, Zhan XM 2011. Enzootic angiostrongyliasis, Guangdong, China, 20082009. Emerg Infect Dis 17: 1335-1336.

Ramirez-Avila L, Slome S, Schuster FL, Gavali S, Schantz PM, Sejvar J, Glaser CA 2009. Eosinophilic meningitis due to Angiostrongylus and Gnathostoma species. Clin Infect Dis 48: 322-327.

Reinhart BJ, Slack FJ, Basson M, Pasquinelli AE, Bettinger JC, Rougvie AE, Horvitz HR, Ruvkun G 2000. The 21-nucleotide let-7 RNA regulates developmental timing in Caenorhabditis elegans. Nature 403: 901-906.

Simon DJ, Madison JM, Conery AL, Thompson-Peer KL, Soskis M, Ruvkun GB, Kaplan JM, Kim JK 2008. The microRNA miR-1 regulates a MEF-2-dependent retrograde signal at neuromuscular junctions. Cell 133: 903-915.

Sokol NS, Ambros V 2005. Mesodermally expressed Drosophila microRNA-1 is regulated by Twist and is required in muscles during larval growth. Genes Dev 19: 2343-2354.

Tsai HC, Lai PH, Sy CL, Lee SS, Yen CM, Wann SR, Chen YS 2011. Encephalitis caused by Angiostrongylus cantonensis after eating raw frogs mixed with wine as a health supplement. Intern Med 50: 771-774.

Tsai HC, Lee SS, Huang CK, Yen CM, Chen ER, Liu YC 2004. Outbreak of eosinophilic meningitis associated with drinking raw vegetable juice in southern Taiwan. Am J Trop Med Hyg 71: 222-226.

van Rooij E 2011. The art of microRNA research. Circ Res 108: 219-234. 
Wallace GD, Rosen L 1966. Studies on eosinophilic meningitis. 2. Experimental infection of shrimp and crabs with Angiostrongylus cantonensis. Am J Epidemiol 84: 120-131.

Wallace GD, Rosen L 1967. Studies on eosinophilic meningitis. IV. Experimental infection of fresh-water and marine fish with $\mathrm{An}$ giostrongylus cantonensis. Am J Epidemiol 85: 395-402.

Wang LC, Chao D, Chen ER 1989. Acquired immunity in rats against Angiostrongylus cantonensis infection. Int $J$ Parasitol 19: 617-620.

Wang LC, Yen CM, Liu CL, Chen ER, Chao D 2012. Effects of age and splenectomy on heavy infection of Angiostrongylus cantonensis in rats. Exp Parasitol 131: 210-214.

Wang QP, Lai DH, Zhu XQ, Chen XG, Lun ZR 2008. Human angiostrongyliasis. Lancet Infect Dis 8: 621-630.

Wienholds E, Kloosterman WP, Miska E, Alvarez-Saavedra E, Berezikov E, de Bruijn E, Horvitz HR, Kauppinen S, Plasterk RH 2005. MicroRNA expression in zebrafish embryonic development. Science 309: 310-311.
Winter AD, Weir W, Hunt M, Berriman M, Gilleard JS, Devaney E, Britton C 2012. Diversity in parasitic nematode genomes: the microRNAs of Brugia pahangi and Haemonchus contortus are largely novel. BMC Genomics 13: 4.

Xu MJ, Liu Q, Nisbet AJ, Cai XQ, Yan C, Lin RQ, Yuan ZG, Song HQ, He XH, Zhu XQ 2010. Identification and characterization of microRNAs in Clonorchis sinensis of human health significance. BMC Genomics 11: 521.

Xu SS, Ni F, Luo DM 2009. Expressed sequence tags (ESTs) analysis of Angiostrongylus cantonensis. Zhongguo Ji Sheng Chong Xue Yu Ji Sheng Chong Bing Za Zhi 27: 248-250.

Xue X, Sun J, Zhang Q, Wang Z, Huang Y, Pan W 2008. Identification and characterization of novel microRNAs from Schistosoma japonicum. PLoS ONE 3: e4034.

Zhao Y, Samal E, Srivastava D 2005. Serum response factor regulates a muscle-specific microRNA that targets Hand 2 during cardiogenesis. Nature 436: 214-220. 
Identification of conserved microRNAs (miRNAs) of Angiostrongylus cantonensis young adults with 50 or more reads

\begin{tabular}{|c|c|c|c|c|}
\hline Family & MiRNAs & Sequence [nucleotides (n)] & Reference miRNAs (accession) & $\begin{array}{l}\text { Reads } \\
\text { (n) }\end{array}$ \\
\hline \multirow[t]{11}{*}{ let-7 } & aca-let-7-1 & UGAGGUAGUAGAUUGUAUAGUU (22) & ppy-let-7f (MIMAT0015726) & 2,674 \\
\hline & aca-let-7-2 & AACUAUACAACCUACUACCUCA (22) & gga-let-7a (MIMAT0001101) & 1,858 \\
\hline & aca-let-7-3 & UGAGGUAGUAGUUUGUGCUGUU (22) & ppy-let-7i (MIMAT0015728) & 832 \\
\hline & aca-let-7-4 & AACCACACAACCUACUACCUCA (22) & gga-let-7b (MIMAT0001102) & 819 \\
\hline & aca-let-7-5 & UGAGGUAGUAGUUUGUACAGUU (22) & ppy-let-7g (MIMAT0015727) & 606 \\
\hline & aca-let-7-6 & CUAUACAAUCUACUACCUCA (20) & hsa-let-7f-5p (MIMAT0000067) & 276 \\
\hline & aca-let-7-7 & UGAGGUAGUAGGUUGUAUGGUU (22) & ppy-let-7c (MIMAT0015723) & 245 \\
\hline & aca-let-7-8 & UGAGGUAGGAGGUUGUAUAGUU (22) & ppy-let-7e (MIMAT0015725) & 217 \\
\hline & aca-let-7-9 & UGAGGUAGUAGGUUGUAUAGU (21) & ngi-let-7 (MIMAT0018413) & 201 \\
\hline & aca-let-7-10 & UGAGGUAGUAGUUUGUGCUGU (21) & gga-let-7i (MIMAT0001098) & 188 \\
\hline & aca-let-7-11 & AGAGGUAGUAGGUUGCAUAGUU (22) & ppy-let-7d (MIMAT0015724) & 79 \\
\hline \multirow[t]{4}{*}{$\operatorname{mir}-1$} & aca-miR-1-1 & UGGAAUGUAAAGAAGUAUGUA (21) & ssc-miR-1 (MIMAT0010187) & 184,946 \\
\hline & aca-miR-1-2 & UGGAAUGUAAAGAAGUAUGUAU (22) & ppy-miR-1 (MIMAT0015720) & 40,263 \\
\hline & aca-miR-1-3 & UGGAAUGUAAAGAAGUAUGU (20) & ppc-miR-1 (MIMAT0011639) & 6,652 \\
\hline & aca-miR-1-4 & UGGAAUGUAAAGAAGUAUGUAG (22) & cte-miR-1 (MIMAT0009502) & 2,196 \\
\hline \multirow[t]{2}{*}{ mir-2 } & aca-miR-2-1 & UAUCACAGCCAGCUUUGAUGU (21) & bma-miR-2a (MIMAT0014096) & 413 \\
\hline & aca-miR-2-2 & UAUCACAGCCAGCUUUGAUGUGC (23) & nvi-miR-2b (MIMAT0015667) & 247 \\
\hline \multirow[t]{2}{*}{$\operatorname{mir}-9$} & aca-miR-9-1 & UCUUUGGUUAUCUAGCUGUAUGA (23) & nlo-miR-9a (MIMAT0018442) & 87 \\
\hline & aca-miR-9-2 & UCUUUGGUUAUCUAGCUGUAUG (22) & ppc-miR-9 (MIMAT0011737) & 60 \\
\hline $\operatorname{mir}-21$ & aca-miR-21-1 & UAGCUUAUCAGACUGAUGUUGA (22) & tgu-miR-21 (MIMAT0014527) & 149 \\
\hline $\operatorname{mir}-29$ & aca-miR-29-1 & UAGCACCAUCUGAAAUCGGUUA (22) & eca-miR-29a (MIMAT0012940) & 167 \\
\hline $\operatorname{mir}-30$ & aca-mir-30-1 & UGUAAACAUCCCCGACUGGAAGCU (24) & bta-mir-30d (MIMAT0003533) & 71 \\
\hline \multirow[t]{2}{*}{ mir-31 } & aca-miR-31-1 & AGGCAAGAUGUUGGCAUAGCU (21) & crm-miR-72 (MIMAT0011567) & 96 \\
\hline & aca-miR-31-2 & AGGCAAGAUGUUGGCAUAGCUGA (23) & bma-miR-72 (MIMAT0014107) & 72 \\
\hline \multirow[t]{4}{*}{$\operatorname{mir}-34$} & aca-miR-34-1 & UGGCAGUGUGGUUAGCUGGUUG (22) & nvi-miR-34 (MIMAT0015682) & 598 \\
\hline & aca-miR-34-2 & GGCAGUGUGGUUAGCUGGUUG (21) & nlo-miR-34 (MIMAT0018435) & 77 \\
\hline & aca-miR-34-3 & UGGCAGUGUGGUUAGCUGGU (20) & aga-miR-34 (MIMAT0005526) & 66 \\
\hline & aca-miR-34-4 & AGGCAGUGUAGUUAGCUGAUUG (22) & bta-miR-34c (MIMAT0003854) & 65 \\
\hline $\operatorname{mir}-44$ & aca-miR-44 & UGACUAGAGACACAUUCAGCU (21) & ppc-miR-45 (MIMAT0011640) & 22,803 \\
\hline \multirow[t]{2}{*}{$\operatorname{mir}-50$} & aca-miR-50-1 & UGAUAUGUCUGGUAUUCUUGGGUU (24) & crm-miR-50 (MIMAT0011589) & 702 \\
\hline & aca-miR-50-2 & UGAUAUGUCUGGUAUUCUUGGG (22) & asu-miR-50 (MIMAT0021461) & 81 \\
\hline $\operatorname{mir}-60$ & aca-miR-60-1 & UAUUAUGCACAUUUUCUAGUUCA (23) & cel-miR-60-3p (MIMAT0000032) & 111 \\
\hline $\operatorname{mir}-67$ & aca-miR-307 & UCACAACCUCCUUGAGUGAG (20) & isc-miR-307 (MIMAT0012701) & 177 \\
\hline \multirow[t]{3}{*}{ mir-71 } & aca-miR-71-1 & UGAAAGACAUGGGUAGUGAGACG (23) & crm-miR-71 (MIMAT0011618) & 92,433 \\
\hline & aca-miR-71-2 & UGAAAGACAUGGGUAGUGAGAU (22) & bfl-miR-71 (MIMAT0009476) & 129 \\
\hline & aca-miR-71-3 & UGAAAGACAUGGGUAGUGAGAUG (23) & api-miR-71 (MIMAT0014735) & 50 \\
\hline $\operatorname{mir}-81$ & aca-miR-81 & UGAGAUCAUCGUGAAAGCUAGU (22) & crm-miR-81 (MIMAT0011570) & 282 \\
\hline \multirow[t]{4}{*}{$\operatorname{mir}-87$} & aca-miR-87-1 & GUGAGCAAAGUUUCAGGUGUG (21) & isc-miR-87 (MIMAT0012707) & 314 \\
\hline & aca-miR-87-2 & GUGAGCAAAGUUUCAGGUGUGC (22) & api-miR-87a (MIMAT0014739) & 141 \\
\hline & aca-miR-87-3 & GUGAGCAAAGUUUCAGGUGU (20) & ame-miR-87 (MIMAT0004434) & 139 \\
\hline & aca-miR-87-4 & GUGAGCAAAGUCUCAGGUGU (20) & bma-miR-87b (MIMAT0014110) & 126 \\
\hline \multirow[t]{3}{*}{ mir-99 } & aca-miR-99-1 & AACCCGUAGAUCCGAACUUGUGU (23) & isc-miR-100 (MIMAT0012683) & 1,783 \\
\hline & aca-miR-99-2 & AACCCGUAGAUCCGAACUUGUG (22) & nvi-miR-100 (MIMAT0015677) & 60 \\
\hline & aca-miR-99-3 & AACCCGUAGAUCCGAACUUGU (21) & tgu-miR-100 (MIMAT0014511) & 50 \\
\hline \multirow[t]{2}{*}{ mir-103 } & aca-miR-103-1 & AGCAGCAUUGUACAGGGCUAUGA (23) & tgu-miR-103 (MIMAT0014523) & 482 \\
\hline & aca-miR-103-2 & AGCAGCAUUGUACAGGGCUAU (21) & gga-miR-103 (MIMAT0001145) & 220 \\
\hline $\operatorname{mir}-124$ & aca-miR-124-1 & UAAGGCACGCGGUGAAUGCCAA (22) & bma-miR-124 (MIMAT0014116) & 129 \\
\hline $\operatorname{mir}-140$ & aca-miR-140-1 & ACCACAGGGUAGAACCACGGA (21) & cfa-miR-140 (MIMAT0006689) & 60 \\
\hline mir-146 & aca-miR-146-1 & UGAGAACUGAAUUCCAUGGGUU (22) & ppy-miR-146a (MIMAT0015767) & 270 \\
\hline $\operatorname{mir}-185$ & aca-miR-185 & UGGAGAGAAAGGCAGUUCCUGA (22) & ppy-miR-185 (MIMAT0015781) & 79 \\
\hline
\end{tabular}




\begin{tabular}{lcrll}
\hline Family & MiRNAs & Sequence [nucleotides (n)] & Reference miRNAs (accession) & $\begin{array}{c}\text { Reads } \\
\text { (n) }\end{array}$ \\
\hline mir-191 & aca-miR-191-1 & CAACGGAAUCCCAAAAGCAGCUG (23) & ppy-miR-191 (MIMAT0015785) & 50 \\
mir-235 & aca-miR-235-1 & UAUUGCACUCGCCCCGGCCUG (21) & crm-miR-235 (MIMAT0011558) & 143 \\
mir-320 & aca-miR-320-1 & AAAAGCUGGGUUGAGAGGGCGA (22) & ppy-miR-320a (MIMAT0015821) & 78 \\
\hline
\end{tabular}

aga: Anopheles gambiae; ame: Apis mellifera; api: Acyrthosiphon pisum; asu: Ascaris suum; bfl: Branchiostoma floridae; bma: Brugia malayi; bta: Bos taurus; cel: Caenorhabditis elegans; cfa: Canis familiaris; crm: Caenorhabditis remanei; cte: Capitella teleta; eca: Equus caballus; gga: Gallus gallus; hsa: Homo sapiens; isc: Ixodes scapularis; ngi: Nasonia giraulti; nlo: Nasonia longicornis; nvi: Nasonia vitripennis; ppc: Pristionchus pacificus; ppy: Pongo pygmaeus; ssc: Sus scrofa; tgu: Taeniopygia guttata. 